\title{
Resistencia a los antimicrobianos en Chile y el paradigma de Una Salud: manejando los riesgos para la salud pública humana y animal resultante del uso de antimicrobianos en la acuicultura del salmón y en medicina
}

\author{
Ana R. Millanao, Carolina Barrientos-Schaffeld, Claudio D. Siegel-Tike, Alexandra Tomova, Larisa Ivanova, \\ Henry P. Godfrey, Humberto J. Dölz, Alejandro H. Buschmann y Felipe C. Cabello
}

\begin{abstract}
Antimicrobial resistance in Chile and The One Health paradigm: Dealing with threats to human and veterinary health resulting from antimicrobial use in salmon aquaculture and the clinic
\end{abstract}

The emergence and dissemination of antimicrobial-resistant bacteria (ARB) is currently seen as one of the major threats to human and animal public health. Veterinary use of antimicrobials in both developing and developed countries is many-fold greater than their use in human medicine and is an important determinant in selection of ARB. In light of the recently outlined National Plan Against Antimicrobial Resistance in Chile, our findings on antimicrobial use in salmon aquaculture and their impact on the environment and human health are highly relevant. Ninety-five percent of tetracyclines, phenicols and quinolones imported into Chile between 1998 and 2015 were for veterinary use, mostly in salmon aquaculture. Excessive use of antimicrobials at aquaculture sites was associated with antimicrobial residues in marine sediments $8 \mathrm{~km}$ distant and the presence of resistant marine bacteria harboring easily transmissible resistance genes, in mobile genetic elements, to these same antimicrobials. Moreover, quinolone and integron resistance genes in human pathogens isolated from patients in coastal regions adjacent to aquaculture sites were identical to genes isolated from regional marine bacteria, consistent with genetic communication between bacteria in these different environments. Passage of antimicrobials into the marine environment can potentially diminish environmental diversity, contaminate wild fish for human consumption, and facilitate the appearance of harmful algal blooms and resistant zoonotic and human pathogens. Our findings suggest that changes in aquaculture in Chile that prevent fish infections and decrease antimicrobial usage will prove a determining factor in preventing human and animal infections with multiply-resistant ARB in accord with the modern paradigm of One Health.

Key words: Resistance, antimicrobials, antibiotics, aquaculture, salmon, One Health.

Palabras clave: Resistencia, antimicrobianos, acuicultura, salmón, Una Salud.
$\mathrm{E}$ 1 surgimiento de bacterias resistentes a los antimicrobianos (BRA) y su diseminación mundial ha sido reconocida como una de las principales amenazas a la salud pública humana y animal del siglo $\mathrm{XXI}^{1-5}$. La relevancia crítica de este problema ha sido ampliamente debatida por organizaciones internacionales y nacionales de salud, gobiernos, fundaciones privadas, sociedades profesionales y, cada vez más, por los consumidores ${ }^{1-6}$. Este último grupo incluye clientes de organizaciones de atención médica y consumidores de alimentos de origen animal y sus derivados, quienes exigen cada vez más que los minoristas distribuyan alimentos certificados que hayan sido producidos de forma sustentable ${ }^{7-9}$.

No es sorprendente que las BRA surjan preferentemente en sitios donde existe un uso masivo y múltiple de antimicrobianos, como hospitales y en la crianza industrial de animales, incluyendo la acuicultura ${ }^{1-3,6,9}$. Durante mucho tiempo se ha reconocido que las infecciones producidas por BRA se asocian con una mayor mortalidad, morbilidad aumentada y un mayor número de complicaciones que conducen a hospitalizaciones prolongadas y a mayores costos de tratamiento ${ }^{1-3,6-12}$. También se ha demostrado repetidamente, en casi todos los países, tanto desarrollados como en desarrollo, que se utilizan muchos más antimicrobianos en la producción de animales para alimento humano y para otros usos veterinarios que en medicina humana ${ }^{2-5}$, uso veterinario que está ampliamente aceptado, aunque juega un papel importante en la aparición de resistencia antimicrobiana en patógenos animales y humanos ${ }^{2,5}$. El paso de antimicrobianos a los ambientes terrestres y acuáticos, como resultado de estas usanzas selecciona bacterias resistentes y aumenta la frecuencia
Universidad Austral de Chile, Valdivia, Chile. Instituto de Farmacia, Facultad de Ciencias (ARM, CBS, CDST, HJD). New York Medical College, Valhalla, New York, USA. Department of Microbiology and Immunology (AT, LI, FCC). Department of Pathology (HPG). Universidad de Los Lagos, Puerto Montt, Chile. Centro i mar and CeBiB (AHB),

Los autores no tienen conflictos de interés que declarar.

Recibido: 6 de febrero de 2018 Aceptado: 27 de febrero de 2018

Correspondencia a: Felipe C. Cabello cabello@nymc.edu 
de variación genética en ellas fomentando la mutación, la recombinación de ADN y la transferencia horizontal de variantes y de nuevos genes de resistencia a antimicrobia$\operatorname{nos}^{1,5,10-14}$. Esta panoplia de variación molecular también facilita la captura de genes de resistencia antimicrobiana nuevos y no reconocidos del resistoma ambiental por patógenos animales y humanos, dificultando aún más la antibioterapia de infecciones ${ }^{1,2,6,12,14,15}$.

Recientemente, el gobierno chileno propuso un esbozo de un plan nacional para prevenir y combatir la resistencia a los antimicrobianos siguiendo las recomendaciones de la Organización Mundial de la Salud (OMS), la Organización de Alimentación y Agricultura (FAO), la Oficina Internacional de Epizootias (OIE) y la Asamblea General de las Naciones Unidas ${ }^{16}$. Hemos participado activamente en el estudio de las cantidades de antimicrobianos utilizados en medicina veterinaria en Chile durante los últimos 15 años, especialmente en la acuicultura del salmón y su posible impacto ${ }^{1,12,17}$. Anteriormente publicamos nuestros hallazgos en numerosos trabajos ${ }^{1,17,18}$, y ahora nos gustaría volver a plantear estos datos, ampliar nuestros hallazgos hasta 2015 y discutir su relevancia para el surgimiento de la resistencia a los antimicrobianos en Chile a la luz del Plan Nacional propuesto ${ }^{1,17,19,20}$.

La determinación de las cantidades de antimicrobianos utilizados en un área determinada, en un período de tiempo bien definido, es la piedra angular para prevenir y combatir la resistencia a antimicrobianos porque los niveles de esta resistencia están directamente relacionados con las cantidades de antimicrobianos utilizados ${ }^{1,10-13}$. Como no se producen antimicrobianos en Chile, todos los antimicrobianos utilizados en este país deben importarse. Entre 1998 y 2015, nuestro análisis indicó que 95\% (7.775 toneladas) de tetraciclinas, fenicoles y quinolonas importadas a Chile

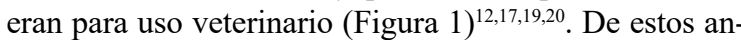
timicrobianos importados, 19\% (1.480 toneladas) fueron quinolonas, $35 \%$ (2.683 toneladas) fueron fenicoles y $46 \%$ (3.612 toneladas) fueron tetraciclinas. De las quinolonas importadas para uso veterinario, se utilizaron 1.132 toneladas $(77 \%)$ en acuicultura. Interesantemente, los fenicoles importados para uso veterinario aumentaron de 3,2 toneladas entre los años 2000-2003 a 1.606 toneladas entre 2012 y 2015, lo que probablemente refleje un mayor uso en acuicultura y más específicamente acuicultura de salmón ${ }^{12,17,18,20}$. Durante este mismo período, se importaron 381 toneladas de antimicrobianos (5\% del total) para uso clínico humano: 312 toneladas $(82 \%)$ de quinolonas, 28 toneladas $(7 \%)$ de fenicoles y 41 toneladas $(11 \%)$ de tetraciclinas. Otra ilustración de la diferencia entre el uso clínico veterinario y humano es que, si bien se importó un promedio de 226 toneladas de tetraciclinas por año para uso veterinario entre 2000 y 2015, sólo se importaron 2,5 toneladas por año, cien veces menos, que para uso humano, en el mismo período ${ }^{12,17,18,20}$. También hemos demostrado que la importación de estos antimicrobianos al país ha sido directamente proporcional a la expansión de la producción de la acuicultura del salmón ${ }^{12,17}$.

Estos hallazgos indican que, como en otros países, el uso de antimicrobianos en animales, específicamente en la acuicultura del salmón en este caso, probablemente impulse la selección de BRA para estos tres grupos altamente importantes de antimicrobianos y también para otros, dado que se usan 20 veces mas antimicrobianos en la salmonicultuta que en medicina humana ${ }^{12,17,18,20}$. Estos hallazgos también indican que antimicrobianos relevantes para medicina humana como son las quinolonas, se han utilizado indiscriminadamente en la medicina veterinaria en Chile, en marcado contraste con las recomendaciones internacionales que indican que el uso veterinario de antimicrobianos útiles en la medicina humana debería restringirse en veterinaria ${ }^{12,17,18,20}$. Un hecho positivo ha sido la reciente disminución del uso de quinolonas altamente efectivas en medicina humana en la acuicultura a raíz de las demandas efectuadas por los reguladores gubernamentales y distribuidores, sugiriendo que el uso de antimicrobianos se puede modificar con relativa rapidez en respuesta a los requerimientos del mercado ${ }^{20}$.

Desafortunadamente, nuestros estudios sugirieron que las cantidades de antimicrobianos que se usarían en medicina veterinaria podrían subestimarse ya que descubrimos que los servicios reguladores gubernamentales responsables de evaluar este uso autorizaron cantidades menores en algunos años que las cantidades realmente importadas para ese uso ${ }^{12,17}$.

Nuestra demostración de la presencia de antimicrobianos residuales en sedimentos marinos, así como de bacterias resistentes a antimicrobianos (BRA) en agua y sedimentos, en áreas a ocho $\mathrm{kms}$ de distancia de las áreas de aplicación, y la presencia de genes de resistencia en estas BRA a los fármacos más utilizados en esta actividad, vale decir: tetraciclinas, fenicoles y quinolonas ${ }^{18}$, es consistente con nuestras conclusiones y las de otros investigadores, que el paso de los antimicrobianos utilizados en la acuicultura chilena al agua dulce y ambientes marinos subyacen a estas observaciones ya que las bacterias en estos ambientes contienen múltiples genes de resistencia a los antimicrobianos ${ }^{18,21}$. Hemos demostrado que las BRA del ambiente marino contienen genes de resistencia a antimicrobianos en elementos móviles como plásmidos e integrones, y que pueden transferir estas estructuras genéticas y sus genes de resistencia a otras bacterias ${ }^{18,22-25}$. Las bacterias marinas y las bacterias patógenas humanas de las regiones acuícolas comparten genes de resistencia a los antimicrobianos y elementos genéticos móviles, sugiriendo que pueden intercambiar estos elementos genéticos mediante la transmisión horizontal de genes, probablemente en el medio acuático ${ }^{18,22-25}$. 


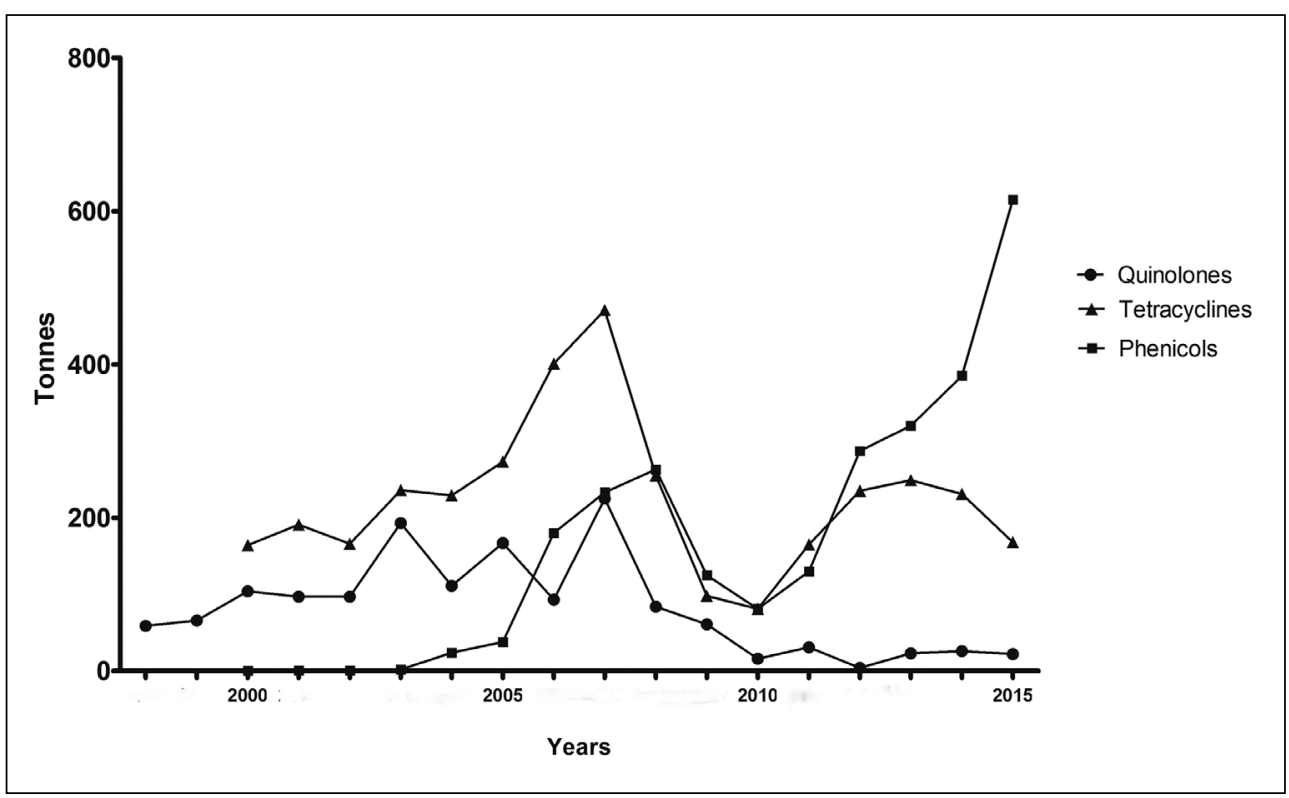

Figura 1. Toneladas métricas de fenicoles, tetraciclinas y quinolonas importadas a Chile para uso en medicina veterinaria, principalmente en acuicultura, 1998-2015. Información obtenida de las referencias $12,17,19$ y 20
Esta línea de investigación ha indicado también que el ambiente marino contiene bacterias que albergan nuevos genes, de resistencia a antimicrobianos que potencialmente pueden pasar a los patógenos de peces y humanos ${ }^{18,23-25}$.

El uso de antimicrobianos en medicina veterinaria, y específicamente en acuicultura, puede tener un impacto negativo en la salud humana al seleccionar BRA en el ambiente acuático y facilitar la transferencia de genes de resistencia a antimicrobianos entre las bacterias marinas y las especies patógenas para los humanos ${ }^{1,12,17,23-25}$. La salud humana también se puede ver afectada por la contaminación de peces silvestres para consumo humano con residuos de antimicrobianos. Estos residuos pueden alterar el microbioma humano ${ }^{26,27}$ y seleccionar BRA en la carne de pescado silvestre y productos acuícolas, factores todos ellos capaces de estimular la transmisión horizontal de genes de resistencia a antimicrobianos ${ }^{1,26,27}$.

Nuestros estudios sugieren de manera importante que las extendidas áreas geográficas utilizadas por la actividad acuícola en Chile son relevantes para la generación de BRA, la diseminación de estas bacterias y de sus genes de resistencia a antimicrobianos a todo el mundo ${ }^{1,11,12,17,18,22-24}$.

El empleo de antimicrobianos en la acuicultura tiene otros impactos en el medio ambiente además de su impacto en la salud humana producido por la selección de BRA y genes de resistencia a los antimicrobianos. Su uso puede disminuir la diversidad biológica en el ambiente con el potencial teórico de facilitar la floración de algas tóxicas y la selección de microorganismos patógenos para el hombre de origen marino resistentes; es el caso de Vibrio parahaemolyticus y patógenos zoonóticos de origen acuático $^{12,28,29}$.
Recientemente se ha descubierto que la diversidad bacteriana en un área de cultivo de salmón es reducida en comparación con la de un sitio de control que carece de actividades de acuicultura de salmón, y pareciera que la función de estas bacterias en el ecosistema también es diferente $^{30}$. La disminución de la diversidad biológica producida por este uso excesivo de antimicrobianos también facilita las infecciones bacterianas de los peces cultivados con nuevos y emergentes patógenos resistentes a antimicrobianos, como Piscirickettsia salmonis, que junto con las floraciones de algas nocivas constituyen una gran amenaza para la industria de la acuicultura ${ }^{1,31,32}$. Por ejemplo, aproximadamente, la mitad de los aislados de P. salmonis en Chile son resistentes a quinolonas, lo que refleja el extenso uso previo de estos terapéuticos en la industria ${ }^{32}$.

Nuestras investigaciones han indicado que el uso indiscriminado de antimicrobianos constituye un riesgo para la salud humana, animal y ambiental, así como para la propia industria de la acuicultura. Por esta razón, propusimos un conjunto de medidas en el año 2011 que podrían implementarse en la industria acuícola chilena por las entidades reguladoras chilenas de sanidad animal y humana para mejorar estos impactos ${ }^{12,17}$. Muchas de nuestras recomendaciones coinciden con las del plan nacional propuesto para prevenir y combatir la resistencia a los antimicrobianos. Sin embargo, nos gustaría sugerir que el plan también debería incorporar medidas basadas en el paradigma de Una Salud para registrar y controlar el uso de antimicrobianos en animales y humanos ${ }^{1,3,33}$. El paradigma Una Salud especifica que la protección de la salud humana se logra mediante la implementación de 
medidas higiénicas en la cría industrial de animales y peces para disminuir el uso de antimicrobianos, porque como nosotros y otros han demostrado, la salud animal y humana están interconectadas y tienen interacciones recíprocas ${ }^{1,3,12,33,34,35}$. Los factores más importantes que facilitan la aparición de BRA y la diseminación de genes de resistencia a antimicrobianos que afectan la salud humana en Chile podrían evitarse mediante una atención veterinaria adecuada a la higiene y el bienestar de los animales, especialmente la de los peces en acuicultura.

Agradecimientos. Este trabajo ha sido apoyado por subvenciones del Lenfest Ocean Program/Pew Charitable Trusts (FCC y AHB), FONDECYT, Núcleo de Investigación (NU02 / 2016, Universidad de Los Lagos y Programa BasalCONICYT FB001) (AHB), y por una beca de la Fundación John Simon Guggenheim (FCC).

\section{Resumen}

El aumento de la resistencia bacteriana múltiple a antimicrobianos es considerado una gran amenaza para la salud pública mundial y como generador de una importante crisis en el funcionamiento de los sistemas de salud. Esta crisis es discutida diariamente por los gobiernos y los parlamentos, las instituciones globales de salud, fundaciones benéficas y de científicos y de profesionales de la salud y también de consumidores de productos animales. En todos los países del orbe se ha identificado al uso de antimicrobianos en la crianza industrial de animales como un importante determinante en la selección de esta resistencia. Aprovechando la oportunidad que se ha planteado en Chile con el diseño del Plan Nacional Contra la Resistencia a los Antimicrobianos, hemos creído importante revisitar y actualizar sumariamente nuestros estudios sobre el uso de antimicrobianos en la acuicultura del salmón y de su potencial impacto en el ambiente y la salud humana y animal. Estos estudios indican que $95 \%$ de tres grupos de antimicrobianos importados al país, que incluyen tetraciclinas, fenicoles y quinolonas, son usados en medicina veterinaria y mayormente en la acuicultura del salmón. Nuestros estudios indican que el excesivo uso de estos antimicrobianos genera la presencia de residuos de antimicrobianos en el ambiente marino hasta $8 \mathrm{~km}$ de los sitios de acuicultura, los que seleccionan a bacterias con resistencia múltiple en dicho ambiente, ya que ellas contienen variados genes de resistencia a estos antimicrobianos. Estos genes de resistencia están contenidos en elementos genéticos móviles incluyendo plásmidos e integrones, los que son trasmitidos a otras bacterias permitiendo su potencial diseminación epidémica entre poblaciones bacterianas. Bacterias del ambiente marino contienen genes idénticos a los genes de resistencia a quinolonas e integrones similares a los de patógenos humanos, sugiriendo comunicación genética entre estas bacterias de diversos ambientes. Alrededor de los recintos de acuicultura, este uso exagerado de antimicrobianos contamina con ellos también a peces silvestres para consumo humano y potencialmente selecciona BRA en su carne y en los productos de acuicultura. El consumo de estos productos selecciona bacterias resistentes en el microbioma humano y facilita también el intercambio genético entre bacterias del ambiente acuático y la microbiota comensal y patógena humana. El pasaje de antimicrobianos al ambiente marino disminuye la diversidad en él, y potencialmente podría facilitar la aparición de florecimientos de algas nocivas, la infección de peces por patógenos piscícolas resistentes los antimicrobianos y la aparición de patógenos zoonóticos resistentes, incluyendo a Vibrio parahaemolyticus. Estos hallazgos sugieren que la prevención de infecciones en peces y la disminución del uso de antimicrobianos en su crianza, será en Chile un factor determinante en la prevención de infecciones humanas y animales con resistencia múltiple a los antimicrobianos, de acuerdo con el paradigma moderno e integral de Una Salud.

\section{Referencias bibliográficas}

1.- Cabello F C, Godfrey H P, Buschmann A H, Dölz H J. Aquaculture as yet another environmental gateway to the development and globalization of antimicrobial resistance. Lancet Infect Dis 2016; 16 (7): e127-e133. Review. PubMed PMID: 27083976.

2.- Allcock S, Young E H, Holmes M, Gurdasani D, Dougan G, Sandhu M S, et al. Antimicrobial resistance in human populations: challenges and opportunities. Glob Health Epidemiol Genom 2017: 2: e4. Review. PubMed PMID: 29276617.

3.- National Academies of Sciences, Engineering, and Medicine, Health and Medicine Division,
Board on Global Health, Forum on Microbial Threats. Combating Antimicrobial Resistance: A One Health Approach to a Global Threat: Proceedings of a Workshop. Washington, DC: National Academies Press (USA); 2017. PubMed PMID: 29227604.

4.- Lekshmi M, Ammini P, Kumar S, Varela M F. The food production environment and the development of antimicrobial resistance in human pathogens of animal origin. Microorganisms 2017; 5(1). pii: E11. PubMed PMID: 28335438.

5.- Venter H, Henningsen M L, Begg S L. Antimicrobial resistance in healthcare, agriculture and the environment: the biochemistry behind the headlines. Essays
Biochem 2017; 61: 1-10. Review. PubMed PMID: 28258225.

6.- Cabello F C, Godfrey H P. Even therapeutic antimicrobial use in animal husbandry may generate environmental hazards to human health. Environ Microbiol 2016; 18: 311-3. PubMed PMID: 26913818.

7.- WHO: Health indicators of sustainable agriculture, food and nutrition security in the context of theRio+20 UN Conference on Sustainable Development. www.who.int/hia/ green_economy/indicators_food.pdf. 2012 (accedido 30 de enero de 2018).

8.- Mie A, Andersen H R, Gunnarsson S, Kahl J, Kesse-Guyot E, Rembiałkowska E, et al. Human health implications of organic food and 
organic agriculture: a comprehensive review. Environ Health. 201; 16 (1): 111. Review. PubMed PMID: 29073935.

9.- Henriksson P J G, Rico A, Troell M, Klinger D H, Buschmann A H, Saksida S, et al. Unpacking factors influencing antimicrobial use in global aquaculture and their implication for management: a review from a systems perspective. Sustain Sci 2017. https://doi. org/10.1007/s11625-017-0511-8 (accessed 2018-01-31).

10.- Cabello F C. Antibióticos y acuicultura. Un análisis de sus potenciales impactos para el medio ambiente y la salud humana y animal en Chile. Análisis de Políticas Públicas. Organización Terram, Publicación No 17, 2003. Santiago, Chile http:// www.terram.cl/docs/App17_Antibioticos_y_ Acuicultura.pdf

11.- Cabello F C. Heavy use of prophylactic antibiotics in aquaculture: a growing problem for human and animal health and for the environment. Environ Microbiol 2006; 8: 1137-44. Review. PubMed PMID: 16817922.

12.- Cabello F C, Godfrey H P, Tomova A, Ivanova L, Dölz HJ, Millanao A, et al. Antimicrobial use in aquaculture re-examined: its relevance to antimicrobial resistance and to animal and human health. Environ Microbiol. 2013; 15: 1917-42. Review. PubMed PMID: 23711078.

13.- Watts J E M, Schreier H J, Lanska L, Hale M $\mathrm{S}$. The rising tide of antimicrobial resistance in aquaculture: sources, sinks and solutions. Mar Drugs 2017;15(6). pii: E158. Review. PubMed PMID: 28587172.

14.- Cabello F C, Godfrey H P. Comment on: Transferable resistance to colistin: a new but old threat. J Antimicrob Chemother 2017; 72: 636-637. PubMed PMID: 27733518.

15.- Cabello F C, Tomova A, Ivanova L, Godfrey H P. Aquaculture and $m c r$ colistin resistance determinants. MBio 2017; 8 (5). pii: e0122917. PubMed PMID: 28974615.

16.- Plan Nacional Contra la Resistencia a los Antimicrobianos. Ministerio de Salud, Chile. 2017. https://urldefense.proofpoint. $\mathrm{com} / \mathrm{v} 2 / \mathrm{url}$ ? $\mathrm{u}=\mathrm{http}-3 \mathrm{~A}$ _diprece.minsal. cl_programas-2Dde-2Dsalud_programas2Denfermedades-2Dtransmisibles_resistencia2Dantimicrobiana_\&d $=$ DwIFAg $\& \mathrm{c}=\mathrm{A} 51$ OX6aSaU1ywwq_3bUC2Q\&r=4DNr_1Pe SVT7RodmQ9igLgkBpXPyAqxHVAHAb $\mathrm{kq}$ asQ\&m=sB3LTVdFP6TIU_hMspHRdJnGl 4Uxtt2SwTUKFY2DWTA\&s $=5$ BPmkphcQEf EWYjlpuKCE9JBvOdEFIybOJRP-fuGIpg\&e= (accessed, 01-30-2018).

17.- Millanao A B, Barrientos H M, Gómez C C, Tomova A, Buschmann A, Dölz H J, et al. Uso inadecuado y excesivo de antibióticos:
Salud pública y salmonicultura en Chile. Rev Med Chile 2011; 139: 107-18. Review. PubMed PMID: 21526325 .

18.- Buschmann A H, Tomova A, López A, Maldonado M A, Henríquez L A, Ivanova L, et al. Salmon aquaculture and antimicrobial resistance in the marine environment. PLoS One. 2012;7(8): e42724. PubMed PMID: 22905164.

19.- Siegel-Tike C S. Estudio cualitativo y cuantitativo de los fenicoles y tetraciclinas importadas y autorizadas para uso y disposición en medicina y en veterinaria en Chile, en el período 2013-2015. Consideraciones sobre su impacto para la salud pública y el medio ambiente. 2016. Tesis. Escuela de Química y Farmacia. Universidad Austral, Valdivia. Chile.

20.- Barrientos-Schaffeld C S. Estudio cualitativo y cuantitativo de las quinolonas y las fluorquinolonas importada y autorizadas para uso y disposición en medicina y en veterinaria en Chile, en el período 2013-2015.

Consideraciones sobre su impacto para la salud pública y el medio ambiente. 2016. Tesis. Escuela de Química y Farmacia. Universidad Austral, Valdivia. Chile.

21.- Miranda C D, Kehrenberg C, Ulep C, Schwarz S, Roberts M C. Diversity of tetracycline resistance genes in bacteria from Chilean salmon farms. Antimicrob Agents Chemother 2003; 47: 883-8. PubMed PMID: 12604516.

22.- Aedo S, Ivanova L, Tomova A, Cabello F C. Plasmid-related quinolone resistance determinants in epidemic Vibrio parahaemolyticus, uropathogenic Escherichia coli, and marine bacteria from an aquaculture area in Chile. Microb Ecol 2014; 68: 324-8. PubMed PMID: 24760167.

23.- Shah S Q, Cabello F C, L'abée-Lund T M, Tomova A, Godfrey H P, Buschmann A H, et al. Antimicrobial resistance and antimicrobial resistance genes in marine bacteria from salmon aquaculture and non-aquaculture sites. Environ Microbiol 2014; 16: 1310-20. PubMed PMID: 24612265

24.- Tomova A, Ivanova L, Buschmann AH, Rioseco M L, Kalsi R K, Godfrey H P, et al. Antimicrobial resistance genes in marine bacteria and human uropathogenic Escherichia coli from a region of intensive aquaculture. Environ Microbiol Rep 2015; 7: 803-9. PubMed PMID: 26259681.

25.- Tomova A, Ivanova L, Buschmann A H, Godfrey H P, Cabello F C. Plasmid-mediated quinolone resistance (PMQR) genes and class 1 integrons in quinolone-resistant marine bacteria and clinical isolates of Escherichia coli from an aquacultural area. Microb
Ecol 2018; 75: 104-112. PubMed PMID: 28642992

26.- Fortt A Z, Cabello F C, Buschmann A H. Residuos de tetraciclina y quinolonas en peces silvestres en una zona costera donde se desarrolla la acuicultura del salmón en Chile. Rev Chilena Infectol 2007; 24: 14-8.

27.- Brinkac L, Voorhies A, Gómez A, Nelson K E. The threat of antimicrobial resistance on the human microbiome. Microb Ecol 2017; 74: 1001-8. Review. PubMed PMID: 28492988.

28.- Hernández C G, Ulloa J, Vergara J A O, Espejo R T, Cabello F C. Infecciones por Vibrio parahaemolyticus e intoxicaciones por algas: problemas emergentes de salud pública en Chile. Rev Med Chile 2005; 133: 1081-8. Review. PubMed PMID: 16311702.

29.- Cabello F C, Espejo R T, Hernández M C, Rioseco M L, Ulloa J, Vergara J A. Vibrio parahaemolyticus O3:K6 epidemic diarrhea, Chile, 2005. Emerg Infect Dis 2007; 13: 655-6. PubMed PMID: 17561569.

30.- Hornick K M, Buschmann A H. Insights into the diversity and metabolic function of bacterial communities in sediments from Chilean salmon aquaculture. 2018. Ann Microbiol 2018; 68: 63-77.

31.- Cabello F C, Godfrey H P. Florecimiento de algas nocivas (FANs), ecosistemas marinos y la salud humana en la Patagonia chilena. Rev Chilena Infectol 2016; 33: 559-560. PubMed PMID: 28112340

32.- Henríquez P, Kaiser M, Bohle H, Bustos $\mathrm{P}$, Mancilla M. Comprehensive antibiotic susceptibility profiling of Chilean Piscirickettsia salmonis field isolates. J Fish Dis 2016; 39: 441-8. PubMed PMID: 26660665.

33.- Boqvist $S$, Söderqvist K, Vågsholm I. Food safety challenges and One Health within Europe. Acta Vet Scand 2018; 60: 1. PubMed PMID: 29298694.

34.- Argudín M A, Deplano A, Meghraoui A, Dodémont M, Heinrichs A, Denis O, et al. Bacteria from animals as a pool of antimicrobial resistance genes. Antibiotics (Basel) 2017; 6 (2). pii: E12. doi: 10.3390/ antibiotics6020012. Review. PubMed PMID: 28587316; PubMed Central PMCID: PMC5485445.

35.- Webb H E, Angulo F J, Granier S A, Scott H M, Loneragan G H. Illustrative examples of probable transfer of resistance determinants from food animals to humans: Streptothricins, glycopeptides, and colistin. F1000Res. 2017 Oct 5;6:1805. doi: 10.12688/ f1000research.12777.1. eCollection 2017. Review. PubMed PMID:29188021; PubMed Central PMCID: PMC5686510. 